Proc. XII Int. School on Theoretical Physics — Symmetry and Structural Properties of Condensed Matter

\title{
Electrical Conductivity of Ethylene Glycol Based Nanofluids with Different Types of Thulium Oxide Nanoparticles
}

\author{
J. FAL ${ }^{a, *}$, A. SidOROWICZ ${ }^{b}$ AND G. ŻYŁA ${ }^{a}$ \\ ${ }^{a}$ Department of Physics and Medical Engineering, Rzeszów University of Technology, Rzeszów, Poland \\ ${ }^{b}$ Institute of Electronic Materials Technology, Warsaw, Poland \\ The paper presents experimental investigation on electrical conductivity of thulium oxides-ethylene glycol \\ $\left(\mathrm{Tm}_{2} \mathrm{O}_{3}-\mathrm{EG}\right)$ nanofluids based on nanoparticles with three different sizes, and prepared in different conditions. \\ Nanofluids were prepared with two-step method with use of the nanoparticles obtained by precipitation method. \\ Measurements were conducted at constant temperature $293.15 \mathrm{~K}$ for various mass concentrations from $0 \%$ to $20 \%$ \\ with $5 \%$ step. The electrical conductivity was measured using conductivity meter MultiLine 3410 (WTW GmBH, \\ Weilheim, Germany) and temperature was stabilized in a water bath MLL 547 (AJL Electronic, Cracow, Poland). \\ The results indicate that increase in mass concentration of nanoparticles in base fluid causes increase in electrical \\ conductivity of $\mathrm{Tm}_{2} \mathrm{O}_{3}-\mathrm{EG}$ nanofluids. The enhancement in electrical conductivity of nanosuspensions of thulium \\ oxide is dependent on particle size.
}

DOI: 10.12693/APhysPolA.132.146

PACS/topics: 74.25.F-, 72.15.Cz, 72.60.+g, 73.63.-b

\section{Introduction}

Nanofluids are created as a suspension of nanoparticles in based fluid. As a based fluid there are commonly used ethylene glycol, deionized water, or oils. Nontypical properties of nanofluids caused that they are hope in many branches of industry. They have great potential in electronic applications, heating buildings and reducing pollution, nuclear system cooling, mechanical, biomedical application [1-3].

Nanosuspensions exhibit very interesting rheological $[4,5]$ and thermal $[6,7]$ properties and these areas are intensively studied. But electrical properties of nanofluids become more and more interesting field. It is also shown that nanofluids may have electrorheological properties $[8,9]$, therefore the study of electrical properties of nanofluids seems to be interesting and relevant.

Electrical conductivity not only brings information on the insulation performance, but also reflects the stability and the degree of dispersion of nanofluid [10]. Despite the increasing interest of researchers of nanofluids are only a few of them interested in electric conductivity of nanofluids. Ganguly et al. [11] measured the effective electrical conductivity of $\mathrm{Al}_{2} \mathrm{O}_{3}$ in water and revealed that these properties are related to both temperature and volume fraction. Konakanchi et al. [12] studied $\mathrm{Al}_{2} \mathrm{O}_{3}, \mathrm{SiO}_{2}$ and $\mathrm{ZnO}$ nanoparticles dispersed in propylene glycol and water. They also confirm the dependence of conductivity on temperature and volumetric concentration. Other researchers are investigating various types of nanoparticles in various base fluids e.g. graphene $[13,14], \mathrm{Pd}$ in water [15], $\mathrm{Pd} / \mathrm{Ag}$ in ethylene glycol and distilled water [16] and $\mathrm{TiO}_{2}$ in ethylene glycol [17].

*corresponding author; e-mail: jacekfal@prz.edu.pl
This paper shows results of studies on electrical conductivity of thulium oxide nanoparticles suspended in ethylene glycol with various mass concentrations.

\section{Materials}

\subsection{Nanoparticles preparation}

Thulium oxide $\left(\mathrm{Tm}_{2} \mathrm{O}_{3}\right)$ nanopowders have been obtained by precipitation method. $\mathrm{Tm}\left(\mathrm{NO}_{3}\right)_{3}$ Alfa Aesar Reacton $99.99 \%$ was used as a raw material for the synthesis of thulium oxide powder. The solutions of thulium nitrate (molar concentration of 0.1 (S1), 0.15 (S2) and 0.25 (S3) M) was dropwise added to intensively stirred solution of the precipitating reagent. Synthesis was carried out using ammonium carbonate (analytic grade, Chempur) dissolved in deionized water at room temperature. The resulting slurries were washed four times with deionized water and ethanol, and dried at $323.15 \mathrm{~K}$ for $24 \mathrm{~h}$. The obtained precursors were calcined in air in a muffle furnace at $1373.15 \mathrm{~K}$ for $2 \mathrm{~h}$.

TABLE I

Properties of thulium oxide $\left(\mathrm{Tm}_{2} \mathrm{O}_{3}\right)$ obtained by precipitation method.

\begin{tabular}{l|c|c|c}
\hline \hline & $\mathrm{S} 1$ & $\mathrm{~S} 2$ & $\mathrm{~S} 3$ \\
\hline molar concentration $[\mathrm{M}]$ & 0.10 & 0.15 & 0.25 \\
temperature of precipittion $[\mathrm{K}]$ & 298.15 & 298.15 & 298.15 \\
crystallite size $[\mathrm{nm}]$ & 58 & 77 & 75 \\
lattice parameter $[\AA]$ & 10.485 & 10.492 & 10.489 \\
surface area $\left[\mathrm{m}^{2} / \mathrm{g}\right]$ & 7.06 & 2.14 & 3.70
\end{tabular}

Table I presents the values of lattice parameters and crystallite sizes calculated from X-ray diffration (XRD) measurements and specific surface area measurements (BET) results of obtained powders. All the obtained 
powders are characterized by a similar lattice constant near $10.49 \AA$. However, one can notice differences in the crystallites sizes. The lowest value of crystallite size $58 \mathrm{~nm}$ was determined for the powder obtained with the use of the $0.1 \mathrm{M}$ thulium nitrate solution (sample $\mathrm{S} 1)$. The increase of molar concentration result in the powders of larger crystallites. Also surface area values depend on the thulium nitrate solution concentration. Figure 1 presents images of $\mathrm{Tm}_{2} \mathrm{O}_{3}$ nanopowders in dry form, pictures were taken with scanning electron microscope CrossBeam Workstation AURIGA (Carl Zeiss, Oberkochen, Germany).
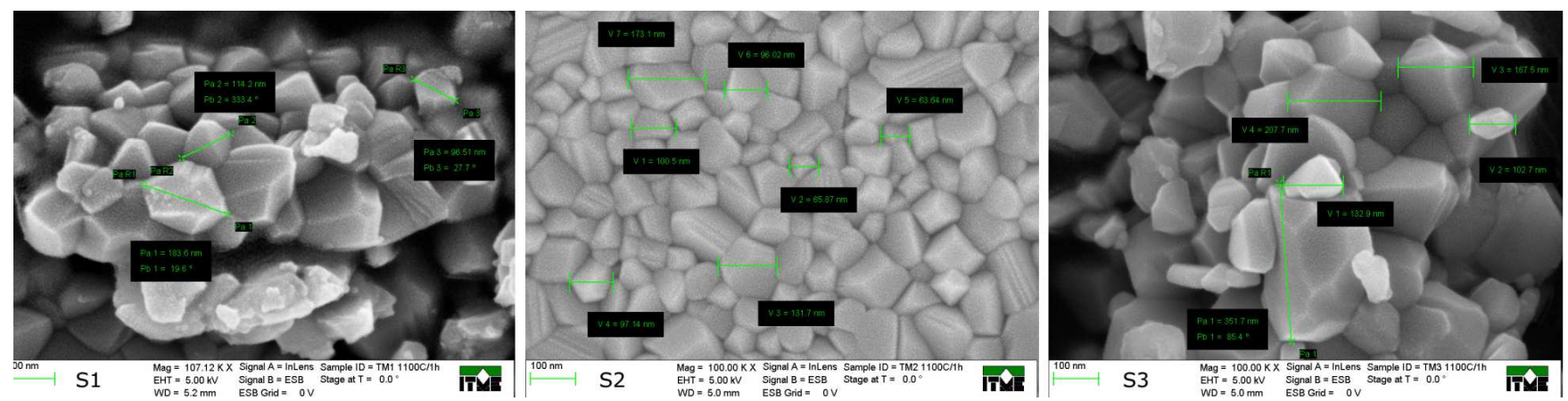

Fig. 1. Scanning electron microscope pictures of dry $\mathrm{Tm}_{2} \mathrm{O}_{3}$ nanoparticles.

\subsection{Sample preparation}

Samples were prepared at mass concentrations from 5 to $20 \mathrm{wt} \%$ with $5 \%$ step. To measure the mass an analytical balance WAS 220/X (Radwag, Radom, Poland) with the accuracy of $0.1 \mathrm{mg}$ was used. The samples were subjected to mechanical stirring for $30 \mathrm{~min}$ in Genius 3 Vortex (IKA, Staufen, Germany), and sonicated for further $200 \mathrm{~min}$ in ultrasoundwave generator Emmi $60 \mathrm{HC}$ (EMAG, Moerfelden-Walldorf, Germany). After preparation of the samples volumetric concentrations were calculated using the formula

$$
\varphi_{v}=\frac{\varphi_{m}}{\rho_{p}\left(\frac{\varphi_{m}}{\rho_{p}}+\frac{1-\varphi_{m}}{\rho_{0}}\right)},
$$

where $\varphi_{v}$ and $\varphi_{m}$ are volume and mass concentration, $\rho_{p}$ and $\rho_{0}$ stands for density of solid particles and base fluid. All nanofluids were prepared at room temperature not exceeding $295.15 \mathrm{~K}$, and studies were carried out immediately after preparation of the samples.

\section{Method}

The electrical conductivity was measured using conductivity meter MultiLine 4310 (WTW GmBH, Weilheim, Germany) with conductivity probe LR 925/01 (WTW GmBH, Weilheim, Germany) with inbuilt automatic temperature compensation. Digital meter allows the simultaneous measure of conductivity and temperature with accuracy $\pm 0.5 \%$ of measured value and $\pm 0.1 \mathrm{~K}$, respectively. The probe was calibrated at conductivity standard KCL $0.01485 \mathrm{~S} / \mathrm{m}$ at $298.15 \mathrm{~K}$ (No. GUM 5.4, Central Office of Measures, Warsaw, Poland).

Electrical conductivity measurements were carried out at $293.15 \mathrm{~K}$ for various volume fraction of nanoparticles in based fluid. The temperature was stabilized using a water bath MLL 547 (AJL Electronic, Cracow, Poland). The temperature was stabilized in water bath for $30 \mathrm{~min}$.
Conductivity value was calculated as the average of 10 measurements taken with every minute.

\section{Results and discussion}

The electrical conductivity of various suspensions based on three different size of thulium oxides nanoparticles and ethylene glycol as base fluid at $293.15 \mathrm{~K}$ were investigated.

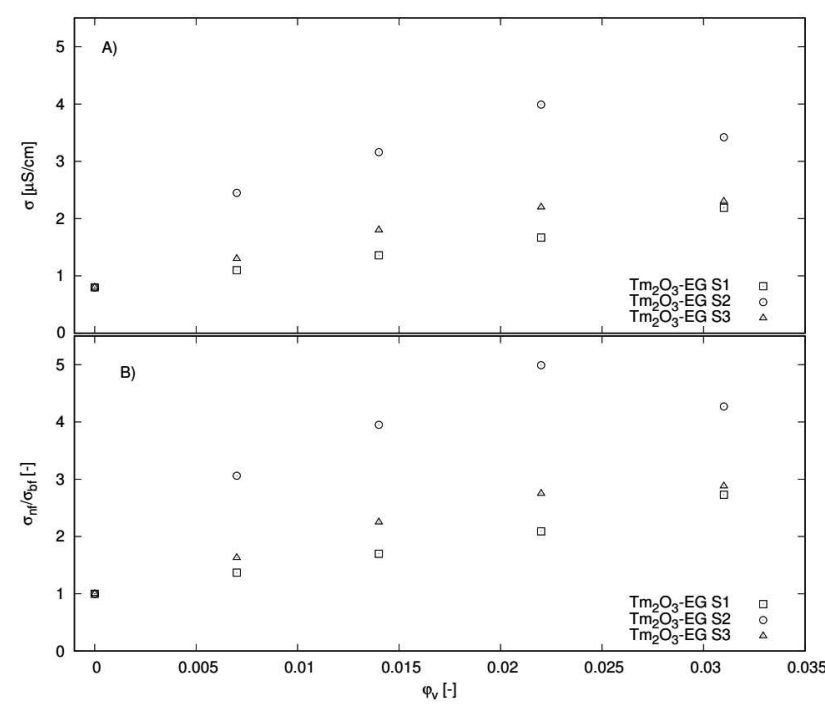

Fig. 2. (A) Dependence of electrical conductivity of TM-EG nanofluids on volume concentration of particles at $293.15 \mathrm{~K}$. (B) Electrical conductivity enhancement of $\mathrm{Tm}_{2} \mathrm{O}_{3}-\mathrm{EG}$ nanofluid at $293.15 \mathrm{~K}$.

The variation of electrical conductivity of suspensions of thulium oxides nanoparticles in ethylene glycol with various size and volume concentration was presented in Fig. 2 and summarized in Table II. Based on the results it is obvious that electrical conductivity of thulium oxides ethylene glycol nanofluids increase with increase of 
TABLE II

Experimental values of the electrical conductivity of $\mathrm{Tm}_{2} \mathrm{O}_{3}$-EG nanofluids for various volume concentrations at $293.15 \mathrm{~K}$.

\begin{tabular}{c|c|c|c|c|c|c|c}
\hline \hline$\varphi_{m}$ & $\varphi_{v}$ & \multicolumn{3}{|c}{$\sigma_{n f}[\mu \mathrm{S} / \mathrm{cm}]$} & \multicolumn{3}{c}{$\sigma_{n f} / \sigma_{b f}$} \\
\cline { 3 - 8 }$[-]$ & {$[-]$} & $\mathrm{S} 1$ & $\mathrm{~S} 2$ & $\mathrm{~S} 3$ & $\mathrm{~S} 1$ & $\mathrm{~S} 2$ & $\mathrm{~S} 3$ \\
\hline 0.00 & 0.000 & 0.80 & 0.80 & 0.80 & 1.00 & 1.00 & 1.00 \\
0.05 & 0.007 & 1.10 & 2.45 & 1.30 & 1.37 & 3.06 & 1.63 \\
0.10 & 0.014 & 1.36 & 3.16 & 1.80 & 1.70 & 3.95 & 2.25 \\
0.15 & 0.022 & 1.67 & 3.99 & 2.20 & 2.09 & 4.99 & 2.75 \\
0.20 & 0.031 & 2.19 & 3.42 & 2.30 & 2.73 & 4.27 & 2.88
\end{tabular}

volume concentrations of nanoparticles in base fluid. For sample S1 increase in electrical conductivity is slight and linear. In case of samples S2 and S3 also there can be observed the same tendency, however above 2.2 vol. $\%$ concentration there is noticeable collapse. Sample S3 also shows a linear increase in electrical conductivity with increase in volume concentrations of nanofluids.

The maximum enhancement was achieved for sample S2 and it was 4.27 times for 3.1 vol.\% concentration.

\section{Conclusion}

The electrical conductivity of various thulium oxide nanofluids was investigated as function of volume concentration at constant temperature. The relation between electrical conductivity and concentration has been shown. Measurements show enhancement in electrical conductivity with increase in concentration of nanoparticles for all cases. The highest enhancement in electrical conductivity was observed for S2 sample. Differences between various thulium oxide nanofluids may be caused by differences in the size of $\mathrm{Tm}_{2} \mathrm{O}_{3}$ nanoparticles crystallite.

\section{References}

[1] G. Huminic, A. Huminic, Renew. Sustain. Energy Rev. 16, 5625 (2012).

[2] N. Putra, Yanuar, F.N. Iskandar, Exp. Therm. Fluid Sci. 35, 1274 (2011).

[3] D. Wen, G. Lin, S. Vafaei, K. Zhang, Particuology 7, 141 (2009).

[4] M. Hojjat, S. Etemad, R. Bagheri, J. Thibault, Int. Commun. Heat Mass 38, 144 (2011).

[5] A.K. Sharma, A.K. Tiwari, A.R. Dixit, Renew. Sustain. Energy Rev. 53, 779 (2016).

[6] S. Thomas, C. Balakrishna Panicker Sobhan, Nanoscale Res. Lett. 6, 377 (2011).

[7] H. Xie, W. Yu, Y. Li, J. Phys. D Appl. Phys. 42, 095413 (2009).

[8] K. Prekas, T. Shah, N. Soin, M. Rangoussi, S. Vassiliadis, E. Siores, J. Coll. Interface Sci. 401, 58 (2013).

[9] T. Brehm, G. Pereira, C.R. Leal, C. Gonsalves, J.P. Borges, M.T. Cidade, Phys. Scr. 90, 035802 (2015).

[10] M. Dong, L.P. Shen, H. Wang, H.B. Wang, J. Miao, J. Nanomater. 2013, 1 (2013).

[11] S. Ganguly, S. Sikdar, S. Basu, Powder Technol. 196, 326 (2009).

[12] H. Konakanchi, R. Vajjha, D. Misra, D. Das, J. Nanosci. Nanotechnol. 11, 6788 (2011).

[13] M. Kole, T. Dey, J. Appl. Phys. 113, 084307 (2013).

[14] M. Hadadian, E.K. Goharshadi, A. Youssefi, J. Nanopart. Res. 16, 2788 (2014).

[15] E.K. Goharshadi, H. Azizi-Toupkanloo, M. Karimi, Microfluid. Nanofluidics 18, 667 (2014).

[16] H. Azizi-toupkanloo, E.K. Goharshadi, P. Nancarrow, Powder Technol. 25, 801 (2014).

[17] J. Fal, A. Barylyak, K. Besaha, Y.V. Bobitski, M. Cholewa, I. Zawlik, K. Szmuc, J. Cebulski, G. Żyła, Nanoscale Res. Lett. 11, 1 (2016). 